This item was submitted to Loughborough's Research Repository by the author.

Items in Figshare are protected by copyright, with all rights reserved, unless otherwise indicated.

\title{
Optimal experimental design for structural health monitoring applications
}

PLEASE CITE THE PUBLISHED VERSION

PUBLISHER

(C) Taylor and Francis

VERSION

AM (Accepted Manuscript)

LICENCE

CC BY-NC-ND 4.0

REPOSITORY RECORD

Lam, H.F., Costas Papadimitriou, and Evangelos Ntotsios. 2019. "Optimal Experimental Design for Structural Health Monitoring Applications”. figshare. https://hdl.handle.net/2134/10789. 
This item was submitted to Loughborough's Institutional Repository (https://dspace.lboro.ac.uk/) by the author and is made available under the following Creative Commons Licence conditions.

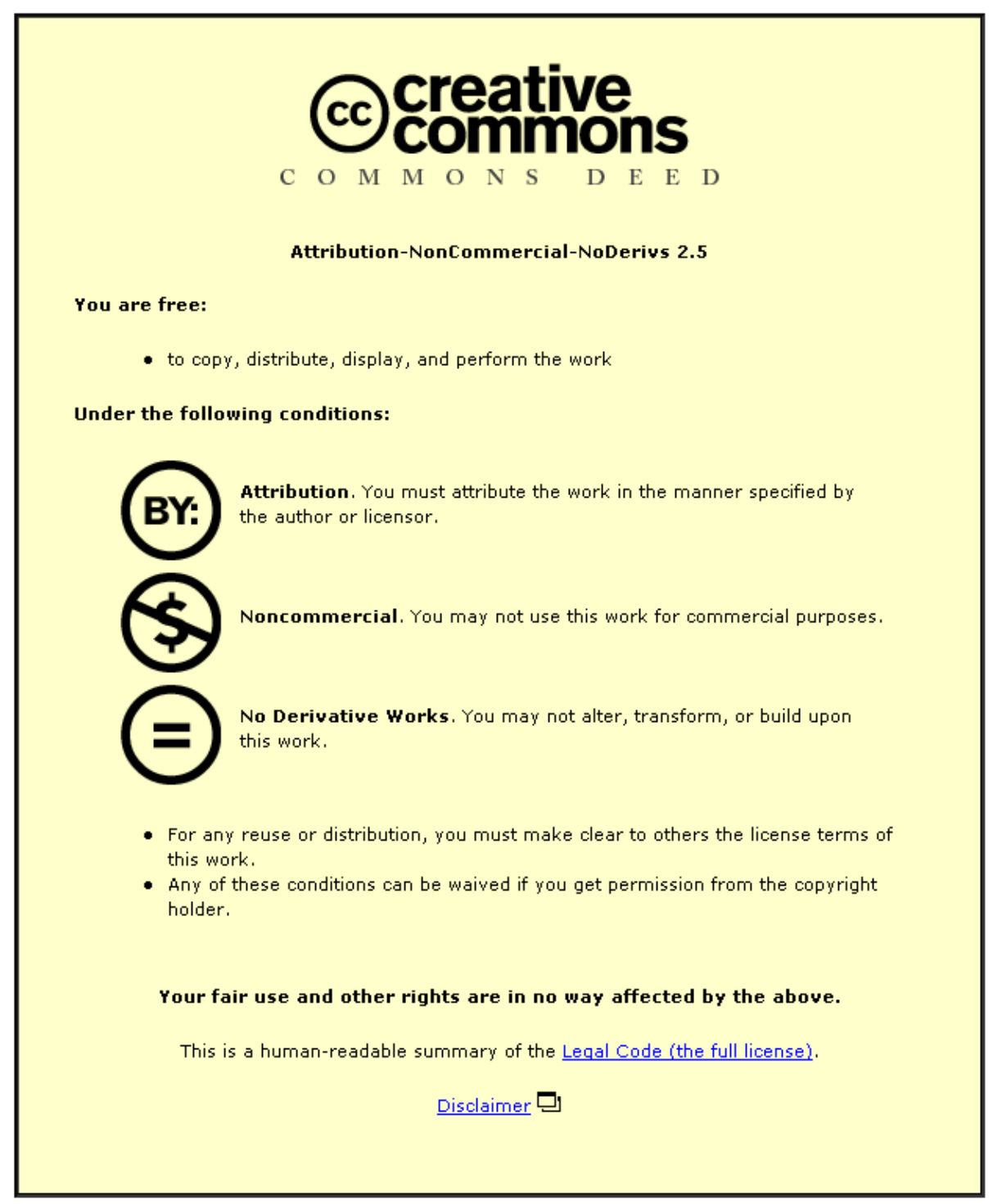

For the full text of this licence, please go to: http://creativecommons.org/licenses/by-nc-nd/2.5/ 


\title{
Optimal experimental design for structural health monitoring applications
}

\author{
H.F. Lam \\ City University of Hong Kong, Department of Building and Construction, Hong Kong, China \\ C. Papadimitriou, E. Ntotsios \\ University of Thessaly, Department of Mechanical \& Industrial Engineering, Volos 38334, Greece
}

\begin{abstract}
Successful structural health monitoring and condition assessment depends to a large extent on the sensor and actuator networks place on the structure as well as the excitation characteristics. An optimal experimental design methodology deals with the issue of optimizing the sensor and actuator network, as well as the excitation characteristics, such that the resulting measured data are most informative for monitoring the condition of the structure. Theoretical and computational issues arising in optimal experimental design are addressed. The problem is formulated as a multi-objective optimization problem of finding the Pareto optimal sensor configurations that simultaneously minimize appropriately defined information entropy indices related to monitoring multiple probable damage scenarios. Asymptotic estimates for the information entropy, valid for large number of measured data, are used to rigorously justify that the selection of the optimal experimental design can be based solely on nominal structural models associated with the probable damage scenarios, ignoring the details of the measured data that are not available in the experimental design stage. Heuristic algorithms are proposed for constructing effective, in terms of accuracy and computational efficiency, sensor configurations. The effectiveness of the proposed method is illustrated by designing the optimal sensor configurations for monitoring damage on a shear model of a building structure.
\end{abstract}

Keywords: Structural identification, experimental design, information entropy, sensor placement, Pareto optima.

\section{INTRODUCTION}

Information theory based approaches (e.g. Shah \& Udwadia 1978, Kammer 1991, Kirkegaard \& Brincker 1994) have been developed to provide rational solutions to several issues encountered in the problem of selecting the optimal sensor configuration. The optimal sensor configuration is taken as the one that maximizes some norm (determinant or trace) of the Fisher information matrix (FIM). Papadimitriou et al. (2000) introduced the information entropy norm as the measure that best corresponds to the objective of structural testing, which is to minimize the uncertainty in the model parameter estimates. An important advantage of the information entropy measure is that it allows one to make comparisons between sensor configurations involving a different number of sensors in each configuration. Furthermore, it has been used to design the optimal characteristics of the excitation (e.g. amplitude and frequency content) useful in the identification of lin- ear and strongly nonlinear models (Metallidis et al. 2003).

The optimal sensor placement strategies depend on the class of mathematical models selected to represent structural behavior as well as the model parameterization within the model class. However, it is often desirable to use the measured data for selecting the most appropriate model class from a family of alternative model classes chosen by the analyst to represent structural behavior. Such classes may be linear (modal models or finite element models), nonlinear elastic or inelastic, each one involving different number of parameters. Model class selection is also important for damage detection purposes for which the location and severity of damage are identified using a family of model classes with each model class monitoring a specific region in a structure (Papadimitriou \& Katafygiotis 2004) or incorporating different mechanisms of damage.

The objective in this work is to optimise the number and location of sensors in the structure such 
that the resulting measured data are most informative for estimating the parameters of a family of mathematical model classes used for structural identification and damage detection purposes. The problem of optimally placing the sensors in the structure is presented for two cases: (i) identification of structural model (e.g. finite element) parameters or modal model parameters (modal frequencies and modal damping ratios) based on acceleration time histories, and (ii) identification of structural model parameters based on modal data. Asymptotic estimates for the information entropy, valid for large number of measured data, are used to rigorously justify that the selection of the optimal experimental design can be based solely on the nominal structural model from a class, ignoring the details of the measured data that are unavailable in the experimental design stage. A heuristic algorithm is used for constructing effective Pareto optimal sensor configurations that are superior, in terms of accuracy and computational efficiency, to the Pareto optimal sensor configurations approximated by genetic algorithms (Zitzler and Thiele 1999), suitable for solving the resulting multi-objective optimization problems. Damage detection results on a shear model of a building are used to illustrate the theoretical developments.

\section{STRUCTURAL IDENTIFICATION AND DAMAGE DETECTION METHODOLOGY}

Consider a parameterized class $M$ of structural models (e.g. a class of finite element models or a class of modal models) chosen to describe the inputoutput behavior of a structure. Let $\theta \in R^{N_{\theta}}$ be the vector of free parameters (physical or modal parameters) in the model class. A Bayesian statistical system identification methodology (Beck \& Katafygiotis 1998, Katafygiotis et al. 1998) is used to estimate the values of the parameter set $\boldsymbol{\theta}$ and their associated uncertainties using the information provided from dynamic test data. For this, the uncertainties in the values of the structural model parameters $\boldsymbol{\theta}$ are quantified by probability density functions (PDF) that are updated using the dynamic test data. The updated PDF is then used for designing the optimal sensor configuration.

According to the Bayesian structural identification methodology, assuming independent and zeromean Gaussian prediction errors $e_{j}(k)$ with variance $\sigma_{j}^{2}$, the updating PDF $p(\boldsymbol{\theta}, \boldsymbol{\sigma} \mid D)$ of the parameter sets $\boldsymbol{\theta}$ and $\boldsymbol{\sigma}=\left(\sigma_{1}, \cdots, \sigma_{N_{o}}\right)$, given the measured data $D$ and the class of models $M$, takes the form (Papadimitriou and Christodoulou 2007):

$$
p(\boldsymbol{\theta}, \boldsymbol{\sigma} \mid D)=\frac{\tilde{c}}{(\sqrt{2 \pi})^{N_{D} N_{0}} \rho(\boldsymbol{\sigma})} \exp \left\{-\frac{N_{D} N}{2} J(\boldsymbol{\theta} ; \boldsymbol{\sigma})\right\} \pi_{\theta}(\boldsymbol{\theta}) \pi_{\sigma}(\boldsymbol{\sigma})
$$

where $J(\boldsymbol{\theta} ; \boldsymbol{\sigma})$ is a measure of fit of the measured response characteristics and the corresponding response characteristics predicted by a particular model in the model class $M$, and $\rho(\boldsymbol{\sigma})$ is a function of the prediction error parameters $\sigma, \pi_{\theta}(\theta)$ and $\pi_{\sigma}(\sigma)$ are the prior distribution for the parameter sets $\boldsymbol{\theta}$ and $\boldsymbol{\sigma}$, respectively, $N=N_{0}, N_{0}$ is the number of response characteristics, $N_{D}$ is the number of measured data sets, and $\tilde{c}$ is a normalizing constant chosen such that the PDF in (1) integrates to one.

For the case for which the response characteristics consist of the response time histories data $D=\left\{\hat{x}_{j}(k \Delta t) \in R^{N_{0}}, j=1, \cdots, N_{0}, k=1, \cdots, N_{D}\right\} \quad$ at $N_{0}$ measured DOFs, where $N_{D}$ is the number of the sampled data using a sampling rate $\Delta t$, then

$J(\boldsymbol{\theta} ; \boldsymbol{\sigma})=\frac{1}{N_{0}} \sum_{j=1}^{N_{0}} \frac{1}{\sigma_{j}^{2}} J_{j}(\boldsymbol{\theta}), \quad J_{j}(\boldsymbol{\theta})=\frac{1}{N_{D}} \sum_{k=1}^{N_{D}}\left[\hat{x}_{j}(k)-x_{j}(k ; \boldsymbol{\theta})\right]^{2}$

$\rho(\boldsymbol{\sigma})=\prod_{j=1}^{N_{0}} \sigma_{j}^{N_{D}}$, and $x_{j}(k ; \boldsymbol{\theta}), j=1, \cdots, N_{d}$, are the predictions of the sampled response time histories obtained from a particular model in the model class $M$ corresponding to a specific value of the parameter set $\boldsymbol{\theta}$.

For the case where the response characteristics consist of modal data $D=\left\{\hat{\omega}_{r}^{(k)}, \hat{\boldsymbol{\phi}}_{r}^{(k)} \in R^{N_{0}}, r=1, \cdots, m, k=1, \cdots, N_{D}\right\}$, where $\hat{\omega}_{r}^{(k)}$ are the modal frequencies and $\hat{\phi}_{r}^{(k)}$ are modeshape components at $N_{0}$ measured DOFs, $m$ is the number of observed modes and $N_{D}$ is the number of modal data sets available, then

$$
J(\boldsymbol{\theta} ; \boldsymbol{\sigma})=\frac{1}{N_{D} N} \sum_{r=1}^{m}\left[\frac{1}{\sigma_{\omega_{r}}^{2}} \sum_{k=1}^{N_{D}} \frac{\left[\omega_{r}(\boldsymbol{\theta})-\hat{\omega}_{r}^{(k)}\right]^{2}}{\left[\hat{\omega}_{r}^{(k)}\right]^{2}}+\frac{N_{0}}{\sigma_{\phi_{r}}^{2}} \sum_{k=1}^{N_{D}} \frac{\left\|\beta_{r}^{(k)} L_{0} \boldsymbol{\phi}_{r}(\boldsymbol{\theta})-\hat{\boldsymbol{\phi}}_{r}^{(k)}\right\|^{2}}{\left\|\hat{\boldsymbol{\phi}}_{r}^{(k)}\right\|^{2}}\right]
$$

where $\omega_{r}(\boldsymbol{\theta})$ and $\phi_{r}(\boldsymbol{\theta}) \in R^{N_{d}}, r=1, \cdots, m$, are the predictions of the modal frequencies and modeshapes obtained for a particular value of the model parameter set $\boldsymbol{\theta}, N=m\left(N_{0}+1\right)$ is the number of measured data per modal set, and $\beta_{r}^{(k)}=\hat{\boldsymbol{\phi}}_{r}^{(k) T}\left(L_{0} \boldsymbol{\phi}_{r}\right) /\left(L_{0} \boldsymbol{\phi}_{r}\right)^{T}\left(L_{0} \boldsymbol{\phi}_{r}\right)$ is a normalization constant that accounts for the different scaling between the measured and the predicted modeshape.

Damage detection is accomplished by introducing a family of $\mu$ model classes $M_{1} \cdots M_{\mu}$ and associating each model class to a damage pattern in the structure, indicative of the location of damage. Each model class $M_{i}$ is assumed to be parameterized by a number of structural model parameters $\boldsymbol{\theta}_{i}$ scaling the stiffness contributions of a "possibly damaged" substructure, while all other substructures are assumed to have fixed stiffness contributions equal to those corresponding to the undamaged structure. Using a Bayesian model selection framework, the probable damage locations are ranked according to the posterior probabilities of the corresponding model 
classes. The most probable model class will be indicative of the substructure that is damaged, while the probability distribution of the model parameters of the corresponding most probable model class will be indicative of the severity of damage in the identified damaged substructure.

Using Bayes' theorem, the posterior probabilities of the various model classes given the data $D$ is

$$
P\left(M_{i} \mid D\right)=\frac{p\left(D \mid M_{i}\right) P\left(M_{i}\right)}{d}
$$

where $p\left(D \mid M_{i}\right)$ is the probability of observing the data from the model class $M_{i}, P\left(M_{i}\right)$ is the prior probability of the model class $M_{i}$, while $d$ is selected so that the sum of all model probabilities equals to one. Assuming there is no prior preference as to what class of models we choose, we may set that $P\left(M_{i}\right)=1 / \mu$ in (4).

The following asymptotic approximation has been introduced to give a useful and insightful estimate of the integral involved in $p(D \mid M)$ in (4) (Papadimitriou and Katafygiotis 2004)

$$
p(D \mid M) \sim(2 \pi)^{N_{\theta} / 2} \frac{\left.\pi_{\theta}(\hat{\boldsymbol{\theta}} \mid M)\left[J_{M}(\hat{\boldsymbol{\theta}}, \hat{\boldsymbol{\sigma}})\right)\right]^{-N_{D} N}}{\sqrt{\operatorname{det} \mathbf{h}_{M}(\hat{\boldsymbol{\theta}}, \hat{\boldsymbol{\sigma}})}}
$$

where, for uniform prior distribution of the parameters $\boldsymbol{\theta}$ in a model class $M, \hat{\theta}$ is the value that minimizes $J_{M}(\hat{\boldsymbol{\theta}}, \hat{\boldsymbol{\sigma}})$, and $\mathbf{h}_{M}(\hat{\boldsymbol{\theta}}, \hat{\boldsymbol{\sigma}})$ is defined by

$$
\mathbf{h}_{M}(\hat{\boldsymbol{\theta}}, \hat{\boldsymbol{\sigma}})=-\left.\nabla_{\theta} \nabla_{\theta}^{T} \ln [J(\boldsymbol{\theta} ; D)]^{-N_{D} N}\right|_{\boldsymbol{\theta}=\hat{\boldsymbol{\theta}}}
$$

in which $\nabla_{\theta}=\left[\partial / \partial \theta_{1}, \cdots, \partial / \partial \theta_{N_{\theta}}\right]^{T}$ is the usual gradient vector with respect to the parameter set $\boldsymbol{\theta}$, and $\hat{\sigma}^{2}$ is the optimal prediction error variance. The approximate estimate is unreliable when the optimal $\boldsymbol{\theta}$ is outside the region $\Theta=\left\{\boldsymbol{\theta}: \boldsymbol{\theta} \leq \boldsymbol{\theta}^{u}\right\}$ of variation of $\boldsymbol{\theta}$, where $\boldsymbol{\theta}^{u}$ are the values of $\boldsymbol{\theta}$ at the undamaged condition. Alternatively, one can use importance sampling method to compute the integral involved in estimating $p(D \mid M)$ in (4) (Papadimitriou and Katafygiotis 2004).

The matrix $\mathbf{h}_{M}(\hat{\boldsymbol{\theta}}, \hat{\boldsymbol{\sigma}})$ in the denominator of (5) is the Fisher information matrix quantifying the uncertainty in the model parameters of the model class $M$ (Papadimitriou et al. 2000). The result in (5) suggests that the selection of the optimal model class among the $\mu$ model classes $M_{1} \cdots M_{\mu}$ depends on the fit each model class provides to the measured data as well as the uncertainty in the parameter values of each model class. The sensor locations are known to affect the value of the information matrix $\mathbf{h}_{M}(\hat{\boldsymbol{\theta}}, \hat{\boldsymbol{\sigma}})$ and the uncertainty in the model parameters of each model class. An optimal sensor configuration strategy should provide informative measurements for the multiple model classes $M_{1} \cdots M_{\mu}$.

\section{OPTIMAL SENSOR LOCATION BASED ON INFORMATION ENTROPY}

The information entropy $H(\boldsymbol{\delta}, D)$ (Papadimitriou et al. 2000), which is a unique scalar measure of the uncertainty in the estimate of the structural parameters $\boldsymbol{\theta}$, is used for optimizing the sensor configuration in the structure for identifying a model class $M$. The information entropy depends on the available data $D$ and the sensor configuration vector $\boldsymbol{\delta}$. It has be shown (Papadimitriou 2004) that for a large number of measured data, i.e. as $N_{D} N \rightarrow \infty$, the following asymptotic result holds for the information entropy for a model class $M$

$$
H_{M}(\boldsymbol{\delta}, D) \sim H_{M}(\boldsymbol{\delta} ; \hat{\boldsymbol{\theta}}, \hat{\sigma})=\frac{1}{2} N_{\theta} \ln (2 \pi)-\frac{1}{2} \ln \left[\operatorname{det} \mathbf{h}_{\mathrm{M}}(\hat{\boldsymbol{\theta}}, \hat{\boldsymbol{\sigma}} ; \boldsymbol{\delta})\right]
$$

where $\hat{\boldsymbol{\theta}} \equiv \hat{\boldsymbol{\theta}}(\boldsymbol{\delta}, D)=\arg \min J(\boldsymbol{\theta} ; D)$ is the optimal value of the parameter ${ }^{\theta}$ set $\boldsymbol{\theta}$ that minimizes the measure of fit $J_{M}(\boldsymbol{\theta} ; D) \equiv J(\boldsymbol{\theta} ; D)$ given in (2) or (3) for a model class $M$, and $\mathbf{h}_{M}(\hat{\boldsymbol{\theta}}, \hat{\boldsymbol{\sigma}}) \equiv \mathbf{h}_{M}(\hat{\boldsymbol{\theta}}, \hat{\boldsymbol{\sigma}} ; \boldsymbol{\delta})$ is an $N_{\theta} \times N_{\theta}$ positive definite matrix defined by (6) and asymptotically approximated by (Papadimitriou 2004)

$$
\mathbf{h}_{M}(\hat{\boldsymbol{\theta}}, \hat{\boldsymbol{\sigma}} ; \boldsymbol{\delta}) \sim \mathbf{Q}_{M}(\boldsymbol{\delta}, \hat{\boldsymbol{\theta}}, \hat{\boldsymbol{\sigma}})
$$

as $N_{D} N \rightarrow \infty$.

For response time history data, substituting (2) into (6) and considering the limiting case $N_{D} N \rightarrow \infty$, the resulting matrix $\mathbf{Q}(\boldsymbol{\delta}, \boldsymbol{\theta})$ appearing in (8) simplifies to a positive semi-definite matrix of the form

$$
\mathbf{Q}_{M}(\boldsymbol{\delta}, \hat{\boldsymbol{\theta}}, \hat{\boldsymbol{\sigma}})=\frac{N_{D}}{2} \sum_{j=1}^{N_{d}} \delta_{j} \frac{1}{\hat{\sigma}_{j}^{2}} \mathbf{P}_{M}^{(j)}(\hat{\boldsymbol{\theta}})
$$

that contains the information about the values of the parameters $\boldsymbol{\theta}$ based on the data from all measured positions specified in $\boldsymbol{\delta}$, while the optimal prediction error variances $\hat{\boldsymbol{\sigma}}^{2}$ are given by $\hat{\boldsymbol{\sigma}}_{j}^{2}=J_{j}(\hat{\boldsymbol{\theta}} ; D)$. The matrix

$$
\mathbf{P}_{M}^{(j)}(\boldsymbol{\theta})=\sum_{k=1}^{N_{D}} \nabla_{\theta} x_{j}(k ; \boldsymbol{\theta}) \nabla_{\theta}^{T} x_{j}(k ; \boldsymbol{\theta})
$$

is a positive semi-definite matrix containing the information about the values of the parameters $\boldsymbol{\theta}$ based on the data from one sensor placed at the $j$-th DOF. The prediction error $\hat{\sigma}_{j}^{2}=J_{j}(\hat{\boldsymbol{\theta}} ; D)$ in (9) is computed from $\hat{\sigma}_{j}^{2}=s_{1}^{2}+s_{2}^{2} g_{j}(\hat{\boldsymbol{\theta}})$, where the first term accounts for constant measurement error and the second term accounts for model error that depends on the strength $g_{j}(\hat{\boldsymbol{\theta}})$ of the response characteristics with the values of $s_{1}^{2}$ and $s_{2}^{2}$ giving the relative size of measurement and model errors. 
For modal data, the resulting matrix $\mathbf{Q}_{M}(\boldsymbol{\delta}, \boldsymbol{\theta})$ simplifies to a positive semi-definite matrix given by

$\mathbf{Q}_{M}(\boldsymbol{\delta}, \boldsymbol{\theta})=\frac{N_{D}}{2} \sum_{r=1}^{m}\left[\frac{\nabla_{\theta} \omega_{r}(\boldsymbol{\theta}) \nabla_{\theta}^{T} \omega_{r}(\boldsymbol{\theta})}{s_{1}^{2}+s_{2}^{2} \omega_{r}^{2}(\boldsymbol{\theta})}+\sum_{j=1}^{N_{d}} \delta_{j} \frac{\nabla_{\theta} L_{0} \phi_{j r}(\boldsymbol{\theta}) \nabla_{\theta}^{T} L_{0} \phi_{j r}(\boldsymbol{\theta})}{s_{1}^{2}+s_{2}^{2}\left\|L_{0} \phi_{r}(\boldsymbol{\theta})\right\|^{2} / N_{0}}\right]$

containing the information about the values of the model parameters $\boldsymbol{\theta}$ based on the modal data from all sensors placed in the structure.

The asymptotic approximation of the information entropy is useful in the experimental stage of designing an optimal sensor configuration. Specifically, the information entropy (7) for a model class $M$ is completely defined by the optimal value $\hat{\boldsymbol{\theta}}$ of the model parameters and the optimal prediction error $\hat{\sigma}_{j}^{2}=J_{j}(\hat{\boldsymbol{\theta}} ; D), j=1, \cdots, N_{0}$, expected for a set of test data, while the time history details of the measured data do not enter explicitly the formulation. The optimal sensor configuration is selected as the one that minimizes the information entropy $(\mathrm{Pa}-$ padimitriou et al. 2000) with respect to the set of $N_{0}$ measurable DOFs. However, in the initial stage of designing the experiment the data are not available, and thus an estimate of the optimal model parameters $\hat{\theta}$ and $\hat{\sigma}$ cannot be obtained from analysis. In practice, useful designs can be obtained by taking the optimal model parameters $\hat{\theta}$ and $\hat{\sigma}$ to have some nominal values chosen by the designer to be representative of the system.

\section{OPTIMAL SENSOR LOCATIONS FOR MUL- TIPLE MODEL CLASSES}

The design of optimal sensor configurations for providing informative measurements for multiple model classes $M_{1}, \ldots, M_{\mu}$ is next addressed. Let $J_{i}(\boldsymbol{\delta}) \equiv I E I_{i}(\boldsymbol{\delta})$ be the effectiveness of a sensor configuration $\delta$ for the $i$ th model class $M_{i}$, where $I E I_{i}(\delta)$ is the information entropy index given by

$$
I E I_{i}(\boldsymbol{\delta})=\frac{H_{i}(\boldsymbol{\delta})-H_{i}\left(\boldsymbol{\delta}_{i, \text { best }}\right)}{H_{i}\left(\boldsymbol{\delta}_{i, \text { worst }}\right)-H_{i}\left(\boldsymbol{\delta}_{i, \text { best }}\right)}
$$

with $H_{i}(\delta) \equiv H_{M_{i}}(\delta ; \hat{\theta}, \hat{\sigma})$. The optimal sensor configuration for the model class $M_{i}$ is selected as the one that minimizes the information entropy index $J_{i}(\boldsymbol{\delta})$. In (12), $\boldsymbol{\delta}_{i, \text { best }}$ is the optimal sensor configuration and $\delta_{i, \text { worst }}$ is the worst sensor configuration for the $i$ th model class. The values of $I E I_{i}(\delta)$ range from zero to one. The most effective configuration corresponds to value of $I E I_{i}(\delta)$ equal to zero, while the least effective configuration corresponds to value of $I E I_{i}(\delta)$ equal to one.

The problem of identifying the optimal sensor locations that minimize the information entropy in- dices for all $\mu$ model classes is formulated as a multi-objective optimization problem stated as follows. Find the values of the discrete-valued parameter set $\delta$ that simultaneously minimizes the objectives (Papadimitriou 2005).

$$
\boldsymbol{J}(\boldsymbol{\delta})=\left(J_{1}(\boldsymbol{\delta}), \ldots, J_{\mu}(\boldsymbol{\delta})\right)
$$

For conflicting objectives $J_{1}(\boldsymbol{\delta}), \ldots, J_{\mu}(\boldsymbol{\delta})$, there is no single optimal solution, but rather a set of alternative solutions, which are optimal in the sense that no other solutions in the search space are superior to them when all objectives are considered. Such alternative solutions, trading-off the information entropy values for different model classes, are known in multi-objective optimization as Pareto optimal solutions. An advantage of the multi-objective identification methodology is that all admissible solutions are obtained which constitute model trade-offs in reducing the information entropies for each model class. These solutions are considered optimal in the sense that the corresponding information entropy for one model class cannot be improved without deteriorating the information entropy for another model class. The optimal points along the Pareto trade-off front provide detailed information about the effectiveness of the sensor configuration for each model class.

An exhaustive search over all sensor configurations for the computation of the optimal sensor configuration is extremely time consuming and in most cases prohibitive. Alternative, genetic algorithms are well suited for performing the multi-objective optimization involving discrete variables. In particular, the strength Pareto evolution algorithm (Zitzler and Thiele 1999) based on genetic algorithms is most suitable for solving the resulting discrete optimization problem and providing near optimal solutions (Papadimitriou 2005).

A more systematic and computationally very efficient approach for obtaining a good approximation of the Pareto optimal front and the corresponding Pareto optimal sensor configurations for a fixed number of $N_{0}$ sensors is to use a sequential sensor placement (SSP) approach (Papadimitriou 2005), extending the SSP algorithm (Papadimitriou 2004) to handle Pareto optimal solutions. The total number of vector function evaluations using the extended SSP algorithm is infinitesimally small compared to the number of vector function evaluations required in an exhaustive search method. Numerical applications (Papadimitriou 2005) show that the Pareto front constructed by this heuristic algorithm, in most cases examined, coincides with, or is very close to, the exact Pareto front. In all cases, the extended SSP algorithm outperforms, in terms of accuracy and computational time, available discrete multi-objective optimization algorithms such as the strength Pareto 
evolution algorithm based on genetic algorithms (Haralampidis et al. 2005).

\section{ILLUSTRATIVE EXAMPLE}

A numerical example is given to illustrate the theoretical developments. Consider a structure represented by a $N_{d}$-DOF chain-like spring-mass model with one end of the chain fixed at the base and the other end free. The nominal values of the stiffness and mass of each link in the chain are chosen to be $k_{i}=k_{0}$ and $m_{i}=m_{0}, i=1, \cdots, N_{d}$, respectively. The nominal model is considered to be the model of the structure at its undamaged condition.

A family of model classes $\left\{M_{12}, M_{34}, M_{56}, M_{78}, M_{9,10}\right\}$ is considered so that each model class $M_{i j}$ is parameterized by two parameters that account for the stiffness values of two out of the ten links. In the selection of the model classes, it was assumed for demonstration purposes that damage is localized in two adjacent links. For each model class the stiffness and mass properties of the links that are not parameterized equal the nominal values of the undamaged structure.

In order to show the influence of the sensor configuration on the prediction effectiveness of the methodology, the case of $N_{0}=3$ sensors is considered. The total number of sensor configurations for this problem is 120 . Among all these configurations, 64 are Pareto optimal sensor configurations for the 5 model classes considered. Among all Pareto optimal sensor configurations, the configuration $\{1,9,10\}$ gives no preference to any model class by providing almost equally informative data for all model classes simultaneously, in the sense that $J_{i}(\delta) \leq J_{\min }$ for all $i=1, \cdots, 5$ with the minimum value of $J_{\min }=0.638$. Among the remaining 63 Pareto optimal sensor configurations, there are configurations $\delta$ that provide more informative data for one or more model classes with $J_{i}(\delta)<0.638$, but simultaneously provide less informative data for other model classes in the family with $J_{i}(\delta)>0.638$. The Pareto optimal sensor configuration $\{1,9,10\}$ is found to provide the most informative data considering all model classes simultaneously.

A damage scenario is considered that corresponds to $40 \%$ stiffness reduction in the first link. Simulated measured modal data are generated by the model with $40 \%$ reduced stiffness at the first link. To simulate the effects of measurement noise and modeling error, $2 \%$ and $5 \%$ noise are respectively added to the modal frequencies and modeshapes simulated by the damaged models. It is expected that the application of the methodology should yield as most probable model class the $M_{12}$, with the value of one of the two parameters predicting the severity of damage in the damaged link. The probability of each model class is obtained by (4). A non- informative prior probability distribution for the parameters of each model is considered.

The results for the probability $P\left(M_{i j} \mid D_{N}\right)$ for all model classes considered are given in Table 1 for the damage scenario considered. The case of $N_{0}=3$ sensors and the case of two and three contributing modes is considered. The measurement locations for $N_{0}=3$ sensors are taken in all cases to be at the optimal locations $\{1,9,10\}$ DOFs of the chain as well as the alternative non-optimal locations $\{8,9,10\}$ and $\{6,8,10\}$. The results are computed using both asymptotic (first row) and importance sampling (second row) method.

It is seen in Table 1 that the effectiveness of the methodology in predicting the location of damage depends on the number of modes and the location of sensors. Specifically, comparing the probabilities in Table 1 for all model classes for the case of three modes $(m=3)$ and for the optimal sensor configuration $\{1,9,10\}$, it is seen that the methodology correctly predicts the location of damage since it gives a probability of one to model class $M_{12}$ and zero probability to all other model classes. Even for $m=2$ modes, the methodology based on importance sampling estimates of the probabilities favors model class $M_{12}$ with high probability 0.99 . However, in the case of $m=2$ modes, the asymptotic approximation provides incorrect predictions of the relative probabilities of the model classes. This is because the estimate of the probability of the model class $M_{78}$ is incorrect due to the fact that the optimal $\hat{\boldsymbol{\theta}}$ is well outside the range $\boldsymbol{\theta} \in\left[0, \theta^{u}\right]$.

Table 1. Asymptotic (first row) and importance sampling (second row) estimates of the probabilities of the model classes.

Sensor Location Sensor Location Sensor Location

$$
\{1,9,10\} \quad\{8,9,10\} \quad\{6,8,10\}
$$

\begin{tabular}{ccccccc}
\hline $\begin{array}{c}\text { Model } \\
\text { Class }\end{array}$ & $m=2$ & $m=3$ & $m=2$ & $m=3$ & $m=2$ & $m=3$ \\
\hline \multirow{2}{*}{$M_{12}$} & 0 & 1.0 & 0 & 0.64 & 0 & 0.75 \\
& 0.99 & 1.0 & 0.62 & 0.64 & 0.62 & 0.73 \\
\hline \multirow{2}{*}{$M_{34}$} & 0 & 0 & 0.55 & 0 & 0.99 & 0 \\
& 0 & 0 & 0 & 0 & 0 & 0 \\
\hline \multirow{2}{*}{$M_{56}$} & 0.01 & 0 & 0 & 0.36 & 0 & 0.25 \\
& 0.01 & 0 & 0.36 & 0.36 & 0.38 & 0.27 \\
\hline \multirow{2}{*}{$M_{78}$} & 1.0 & 0 & 0.45 & 0 & 0.01 & 0 \\
& 0 & 0 & 0.02 & 0 & 0 & 0 \\
\hline \multirow{2}{*}{$M_{910}$} & 0 & 0 & 0 & 0 & 0 & 0 \\
& 0 & 0 & 0 & 0 & 0 & 0 \\
\hline
\end{tabular}

Using the other two non-optimal sensor configurations $\{8,9,10\}$ and $\{6,8,10\}$, the methodology correctly predicts the location of damage but with significantly lower probability than the one obtained for the case of sensor configurations $\{1,9$, $10\}$. This is due to the lack of significant information for updating model class $M_{12}$ provided by the non-optimal sensor configurations. Besides favoring 
model class $M_{12}$ with probability 0.64 (for sensor configuration $\{8,9,10\}$ ) or 0.73 (for sensor configuration $\{6,8,10\})$, the methodology also favors the model class $M_{56}$ with probability 0.36 or 0.27 , respectively. This is indicative of the fact that the reliability of the predictions depend on the location of sensors in the structure which affects the amount of information contained in the data for updating each model class in the family of model classes. Note that the reliability of predictions deteriorates for the case of $m=2$ modes.

It should be noted that for all three sensor configurations and for $N=3$ modes, the asymptotic and importance sampling estimates give qualitatively similar prediction for the location of damage. However, this is not true for the case of $N=2$ modes, where the asymptotic estimates may give false results, mainly due to the fact that the optimal $\hat{\boldsymbol{\theta}}_{i}$ is outside the range $\boldsymbol{\theta}_{i} \in\left[0, \boldsymbol{\theta}_{i}^{u}\right]$.

The most probable values of the parameter set of the most probable model class for all cases predicting the location of damage were also obtained and shown to be close to $60 \%$ and $100 \%$ of the nominal values of the undamaged models, thus predicting close to $40 \%$ stiffness reduction in the lowest link which suggests that the severity of damage is also correctly identifying the optimal model class.

\section{CONCLUSIONS}

The methodology proposed in this study is useful for designing optimal sensor configurations that provide the most informative data for identifying the parameters of a family of model classes introduced for damage detection purposes. Information entropy indices were introduced to measure the quality of information contained in the measured data. An asymptotic estimate, valid for large number of data, was used to justify that the sensor placement design can be based solely on a nominal model, ignoring the details in the measured data. Analytical expressions and numerical results demonstrated that the design of the optimal sensor configuration also depends on model and measurement error assumptions. The optimal sensor location problem for identifying the parameters of multiple model classes is formulated as a multiple objective optimization problem. Heuristic algorithms, available for solving the optimal sensor location problem for a single or multiple model classes, are superior, in terms of accuracy and computational efficiency, to genetic algorithms suitable for solving the resulting multi-objective optimization problem.

\section{ACKNOWLEDGEMENTS}

The work described in this paper was substantially supported by a grant from the Research Grants Council of the Hong Kong Special Administrative Region, China (CityU 114706). Also, it was cofunded $75 \%$ from the European Union (European Social Fund) and 25\% from the Greek Ministry of Development (General Secretariat of Research and Technology) and the private sector, under grant 03E $\Delta-524$ (PENED 2003).

\section{REFERENCES}

Beck, J. L. \& Katafygiotis, L. S. 1998. Updating Models and their Uncertainties - Bayesian Statistical Framework. Journal of Engineering Mechanics (ASCE), 124(4):455461.

Christodoulou K. \& Papadimitriou, C. 2007. Structural Identification based on Optimally Weighted Modal Residuals. Mechanical Systems and Signal Processing, 21, 4-23.

Kammer, D.C. 1991. Sensor Placements for on Orbit Modal Identification and Correlation of Large Space Structures. Journal of Guidance, Control and Dynamics, 14:251-259.

Katafygiotis, L. S., Papadimitriou C. \& Lam, H. F. 1998. A Probabilistic Approach to Structural Model Updating. International Journal of Soil Dynamics and Earthquake Engineering, 17(7-8):495-507.

Kirkegaard, P.H. \& Brincker, R. 1994. On the Optimal Locations of Sensors for Parametric Identification of Linear Structural Systems. Mechanical Systems and Signal Processing, 8:639-647.

Metallidis, P., Verros, G., Natsiavas, S. \& Papadimitriou, C. 2003. Identification, Fault Detection and Optimal Sensor Location in Vehicle Suspensions. Journal of Vibration and Control, 9(3-4):337-359.

Papadimitriou, C. 2004. Optimal Sensor Placement Methodology for Parametric Identification of Structural Systems. Journal of Sound and Vibration, 278(4):923-947.

Papadimitriou, C. 2005. Pareto Optimal Sensor Locations for Structural Identification. Computer Methods in Applied Mechanics and Engineering, 194(12-16):1655-1673.

Papadimitriou, C., Beck, J. L. \& Au, S. K. 2000. EntropyBased Optimal Sensor Location for Structural Model Updating. Journal of Vibration and Control, 6(5):781-800.

Papadimitriou, C. \& Katafygiotis, L.S. 2004. Bayesian Modeling and Updating. Engineering Design Reliability Handbook, E. Nikolaidis, D.M. Ghiocel and S. Singhal (Eds), CRC Press.

Shah, P. \& Udwadia, F.E. 1978. A Methodology for Optimal Sensor Locations for Identification of Dynamic Systems. Journal of Applied Mechanics, 45:188-196.

Zitzler, E. \& Thiele, L. 1999. Multiobjective Evolutionary Algorithms: A Comparative Case Study and the Strength Pareto Approach. IEEE Transactions on Evolutionary Computation, 3(4):257-271. 